\title{
El impacto del programa del 0,52 del IRPF en las organizaciones de acción social
}

\author{
Vicente Marbán Gallego
}

\begin{abstract}
Resumen: El programa de asignación tributaria del 0,52\% de la cuota íntegra del Impuesto sobre la Renta de las Personas Físicas (IRPF en adelante) desde su inicio jurídico con la Ley de Presupuestos Generales del Estado 33/1987 y su puesta en práctica en la convocatoria del IRPF de 1989, sigue vigente en la actualidad y ha transcurrido en paralelo a la expansión de las entidades del Tercer Sector de acción social desde inicios de los años 90. El programa del 0,52 (0,7\% desde convocatoria 2009) con sus más de 20 años de funcionamiento y su singularidad como programa público de ámbito nacional además de fortalecer el Tercer Sector de Acción Social y las sinergias entre el sector público y el sector social ha contribuido al desarrollo de las entidades sociales especialmente en su vertiente más prestacional. Para reflejar tal desarrollo empezamos con una aproximación histórico-institucional del programa analizando los cambios más relevantes en sus principios básicos. Seguidamente analizamos la evolución de entidades, programas e importes concedidos desde su creación. Finalmente, nos centramos en el impacto socioeconómico del programa del 0,52 en las entidades sociales sin olvidar las limitaciones de las que todavía adolece dicho programa.
\end{abstract}

Palabras clave: Intervención social, Tercer Sector de acción social, impacto socioeconómico, ONG, voluntariado.

\section{INTRODUCCIÓN}

El auge de las organizaciones del Tercer Sector y en particular de las de acción social en su vertiente más prestacional es un hecho desde principios de los años 90. El contexto sociopolítico ha sido favorable para su expansión gracias a la revitalización de la sociedad civil española, a la reestructuración del Estado de Bienestar (descentralización de la provisión y contención presupuestaria), y a la emergencia de nuevos riesgos sociales (inmigración, envejecimiento y cambios en las estructuras familiares). Estos factores han confluido en la necesidad de organizaciones sociales 
que canalicen voluntariado (sociedad civil) y que descongestionen, abaraten y flexibilicen la provisión directa de servicios sociales por parte de las distintas administraciones del Estado. El programa del 0,52 (que desde la declaración de la renta a realizar en 2009 para el ejercicio fiscal de 2008 será del 0,7\%1) con sus años 21 años de existencia y su singularidad como programa público de ámbito nacional en parte ha contribuido a fortalecer el Tercer Sector de Acción Social (TSAS en adelante) y a crear sinergias entre el sector público y el sector social, entre el ámbito estatal y el ámbito territorial de las políticas sociales.

En este contexto, es nuestra intención empezar con una aproximación históricoinstitucional del programa y de su eficacia como política de igualdad analizando los cambios más relevantes en lo relativo a sus principios básicos. Seguidamente, veremos como el desarrollo del 0,52 lo ha sido también de las propias entidades de acción social y viceversa, analizando para ello la evolución de entidades, programas e importes concedidos. Finalmente, nos centraremos en el impacto socioeconómico del programa del 0,52 en las entidades sociales sin olvidar las limitaciones de las que todavía adolece dicho programa.

\section{ELEMENTOS DE CAMBIO Y DE PERMANENCIA EN EL PROGRAMA DEL 0,52 DEL IRPF}

La historia del programa del 0,52\% se inicia desde el punto de vista jurídico con la Ley de Presupuestos Generales del Estado 33/1987, de 23 de diciembre, por el que se establece la asignación tributaria del 0,524\% de la cuota íntegra del IRPF bien a la Iglesia Católica o bien a “otros fines de interés social”. Posteriormente con el RD 825/1988, de 15 de julio se regularían los "fines de interés social” de la asignación tributaria del IRPF y el RD 195/1989, de 17 de febrero, donde se establecen los requisitos y procedimientos para solicitar las correspondientes subvenciones, si bien ambos fueron modificados por el RD 223/1991, de 22 de febrero.

Estos Decretos sentaron las bases de las sucesivas convocatorias que se han ido realizando desde 1989 hasta la actualidad, definiendo una serie de requisitos básicos para que las entidades puedan optar a dichas subvenciones, así como los criterios de valoración de las entidades y de los programas solicitados.

Los requisitos para las entidades solicitantes han permanecido prácticamente inalterables durante estos años y se sintetizan en los siguientes aspectos:

1 La elevación del 0,52\% al 0,7\% se contempla por primera vez en la Ley 42/2006, de 28 de diciembre, de Presupuestos Generales del Estado para el año 2007. No obstante, en la práctica esta elevación se aplicará desde la convocatoria de 2009 para el ejercicio fiscal de 2008. 
a) Estar legalmente constituidas.

b) Tener ámbito estatal o que propongan programas a ejecutar en sus respectivos ámbitos territoriales relacionados con los programas de interés general determinados en las correspondientes convocatorias.

c) Carecer de fines de lucro. Esto no excluye a aquellas que desarrollen actividades de carácter comercial siempre que los beneficios se inviertan totalmente en los fines sociales previstos en el RD 195/1989.

d) Tener como fines institucionales principales la realización de alguna/a actividad/es reflejada/s en la correspondiente convocatoria.

e) Acreditar que se hayan al corriente de las obligaciones tributarias y de la Seguridad Social.

f) Haber justificado suficientemente las ayudas económicas recibidas con anterioridad del Ministerio de Trabajo y Asuntos Sociales.

g) Disponer de la estructura suficiente para garantizar el cumplimiento de sus objetivos acreditando la experiencia operativa suficiente para ello.

En cuanto a los criterios por los que se valora a las entidades solicitantes así como a los programas solicitados, tampoco han variado sustancialmente durante la trayectoria de convocatorias entre 1989 y el 2008, salvo en los requisitos adicionales que tienen que cumplir desde 2005 aquellas que soliciten financiación para programas integrales plurianuales dirigidos a las personas en situación o en riesgo de exclusión social (haber sido subvencionadas en la convocatoria anterior por lo menos para 8 colectivos de atención, estar constituidas legalmente al menos 10 años antes de la fecha de publicación de la convocatoria, y que el 75\% del personal participante en el desarrollo de los programas tenga un contrato de trabajo indefinido).

En lo que respecta a las entidades, el núcleo principal de criterios para su valoración en cuanto a los programas no plurianuales, que representaron en la Convocatoria 2007 el 99,8\% del total de programas subvencionados (González y de Cabo, 2008), apenas ha cambiado durante ese periodo primándose especialmente el alcance o el grado de implantación de la entidad, una antigüedad anterior a la convocatoria superior a dos años, su grado de especialización, su estructura y capacidad financiera y de gestión, el número de voluntarios o el grado de cumplimiento de la entidad en las subvenciones concedidas en convocatorias anteriores. No obstante, a medida que han ido avanzando las convocatorias estas se han ido adaptando a los tiempos. Por un lado, obviamente han desaparecido criterios antaño relevantes como la existencia de convenios entre la entidad solicitante y los Ministerios de Justicia e Interior para realizar la extinta prestación social. Por otra parte, otros criterios se han ido adaptando a una realidad donde se exige a las ONG cada vez más eficacia y transpa- 
rencia en la gestión, mayor diversificación en sus ingresos, especialmente en un contexto de restricciones presupuestarias, y un papel más activo y ejemplarizante en la lucha contra la exclusión sociolaboral. Por ejemplo, desde entrada la mitad de los años 90 se pasó de primar genéricamente a las entidades que "presentasen una diversificación de fuentes de financiación"² a hacerlo con aquellas cuya financiación privada representase al menos, el 10\% de sus ingresos. En los últimos años también se han empezado a valorar positivamente aspectos relacionados con el control de calidad, como la realización de Auditorías externas o contar con sistemas de evaluación y calidad, así como otras cuestiones relacionadas con una mayor cohesión social en perspectiva intrasociativa. En particular, se tiende a primar a aquellas entidades cuyos criterios de contratación favorezcan la integración laboral de personas con discapacidad o la de grupos sociales sobre los que existen medidas especiales de fomento del empleo.

Los criterios por los que se valoran los programas solicitados apenas se han visto alterados en el periodo analizado salvo la obvia desaparición del criterio de la participación de objetores de conciencia en los programas a subvencionar. Dichos criterios en esencia suelen giran en torno a cuatro variables: el fomento del trabajo en redes y plataformas, la corresponsabilidad en la financiación del programa, la coherencia entre objetivos y medios técnicos y humanos, y la continuidad de los programas valorados positivamente en convocatorias anteriores. En concreto, y dependiendo también de la experiencia de la entidad en la gestión de programas sociales, tiende a darse prioridad a los programas que sean cofinanciados por la propia entidad, por las CCAA en las que se desarrollan los programas o por la Unión Europea. Igualmente, la amplitud del ámbito territorial que abarca el programa, el presupuesto presentado y el coste medio del usuario, los objetivos y número de beneficiarios o el número de voluntarios que colaboran en el programa se valoran positivamente en la concesión de subvención al programa solicitado.

Quizás los cambios más relevantes que se ha producido en la trayectoria del 0,52\% atañe a la ampliación al 0,7\% para la convocatoria del 2009 (ejercicio fiscal 2008), a la mencionada implantación en el año 2005 de una nueva modalidad de financiación a través de los programas plurianuales (González y de Cabo, 2008) y al sistema de distribución del porcentaje del 0,52 que se introdujo en la Ley de Presupuestos Generales del Estado del 2000 previo acuerdo firmado el 24 de septiembre de 1999 entre la Plataforma de ONG de acción social y el Gobierno. Hasta entonces tan solo se podía elegir dos opciones para la distribución del 0,52: el sostenimiento de la Iglesia Católica u "otros fines de interés social". Desde dicho acuerdo, se incorporaron fundamentalmente tres novedades:

a) La posibilidad de marcar también ambos destinos simultáneamente en cuyo caso se destinaría el 1,04\% de la cuota íntegra del IRPF. 
b) Los fondos de las declaraciones en las que los ciudadanos no marcasen ninguna casilla retornarían a los Presupuestos Generales del Estado y no a fines sociales como ocurría antes.

c) Dado que, como después veremos, este acuerdo empezaría a notarse en una reducción del importe recaudado a partir de la convocatoria de subvenciones del IRPF del 2001, también se acordó que, para compensar la previsible pérdida de recursos destinados a "otros fines de interés social" se garantizaría en los Presupuestos Generales del Estado una cantidad mínima para estos fines. Si la recaudación del IRPF para fines de interés social fuese inferior a esta cantidad el Ministerio de Economía y Hacienda aportaría el resto. En concreto, para los ejercicios fiscales de 1999 y 2000 (es decir, para las convocatorias de subvenciones del IRPF 2001 y 2002) la cantidad mínima se fijó en 19.000 millones de pesetas (114,2 millones de euros aproximadamente) y en la convocatoria de 2005 fue de 123,6 millones de euros. El resultado fue que a pesar de las campañas de llamamiento a la ciudadanía para que decidieran sobre el destino del 0,52, hasta el 2005 los importes recaudados fueron inferiores a la cantidad mínima establecida. Sirva de ejemplo, como para la convocatoria de subvenciones del 2004 se recaudaron 115,2 millones de euros para "otros fines de interés social" (campaña del IRPF 2002) de los 118,8 que se establecieron como cantidad mínima para esa convocatoria con lo que los 3,5 millones restantes los aportó el Ministerio de Economía y Hacienda.

En definitiva, sucede que tras los requisitos analizados anteriormente, en realidad, subyacen una serie de principios que han ido permaneciendo con mayor o menor éxito durante el funcionamiento del 0,52 a lo largo de estos 21 años y que dan sentido a este programa. En concreto nos referimos a principios como:

- Solidaridad interterritorial y cohesión social. Entre los requisitos y criterios de valoración de las entidades solicitantes se deja entrever cómo la lógica redistributiva de este programa atiende al principio de redistribución y compensación de territorios según las necesidades reales y la atención a todos los colectivos. Para ello se prima especialmente el alcance o grado de implantación de la entidad, sobre todo a aquellas entidades que desarrollan su acción preferentemente en varias CC.AA. Con ello, además, se fortalecen las redes organizativas entre las ONG de acción social y su agrupación en federaciones y confederaciones con el fin de abarcar una serie de necesidades que no se detienen en las fronteras regionales. En otras palabras, el 0,52\% se concibe como un programa supraterritorial creado sobre la base de un impuesto de carácter estatal recaudado y dirigido hacia colectivos con necesidades también supraterritoriales.

- Transversalidad de programas y colectivos de atención. Este principio se refleja en la amplia variedad de programas que abarca y en la transversalidad de las necesidades de una serie de colectivos que pueden llegar a ser beneficiarios de distintos programas simultáneamente. 
- Adaptabilidad: El hecho de que los programas se elaboren previa consulta con la Conferencia Sectorial de Asuntos Sociales y especialmente con la Comisión Permanente del Consejo Estatal de Organizaciones No Gubernamentales de Acción Social, acerca a las administraciones públicas a la realidad cotidiana de los colectivos más precarios permitiendo la adaptación del programa del 0,52 a las necesidades y colectivos emergentes en cada convocatoria del IRPF.

- Complementariedad. Especialmente con otras fuentes de financiación públicas y privadas que aun siguiendo una lógica redistributiva diferente o más localizada puedan atender a colectivos precarios.

\section{EL PROGRAMA DEL 0,52 Y LA CONSOLIDACIÓN DEL TERCER SECTOR DE ACCIÓN SOCIAL}

En cierta manera el 0,52 surgió a finales de los años 80 como un reflejo de la administración pública a sus propios déficit de cobertura ante unas demandas ciudadanas crecientes cuyas necesidades latentes en realidad ya estaban siendo anticipadas por unas entidades de acción social a las que en muchos casos la falta de recursos no les alcanzaba para cubrir dichas necesidades.

Entrados los años 90, el 0,52 fue adquiriendo cada vez mayor significación a medida que se consolidaba un clima social favorable hacia una mayor responsabilidad de la sociedad civil en la provisión del bienestar en un contexto en el que la ciudadanía parecía aceptar los fines pero cuestionaba los medios del Estado de Bienestar. Este factor, unido a la proximidad de las entidades no lucrativas a los ciudadanos más precarios acabó reforzando el papel de las ONG convirtiéndolas en un medio legitimado para llevar a buen puerto una parte de los fondos públicos como ocurre con el 0,52. Una legitimidad social que parece estar avalada por las encuestas (las ONG son consideradas como realmente necesarias por el $63 \%$ de los encuestados) y que se refuerza con una visibilidad social cada vez mayor (el $88 \%$ confirma que las conoce) 3 .

Analizando la historia en cifras del 0,52 es fácil comprobar como su evolución en realidad se asemeja a la del propio Tercer Sector. De hecho, el sustancial incremento de las entidades solicitantes y de los programas solicitados en el 0,52 entrados los años 90 es un reflejo del espectacular crecimiento del Tercer Sector de acción social y de las necesidades de atención detectadas por estas en esos años tal y como han puesto de manifiesto numerosos investigadores y trabajos al respecto: Rodríguez Cabrero y Montserrat (1996); Salinas, Rubio y Cerezo (2001); Plataforma para la Promoción del Voluntariado en España (V.AA., 1997); Fundación Tomillo (V.AA., 2000); Fundación BBVA (Salamon et al., 2001); Pérez Díaz y López Novo (2003); y Fundación FOESSA (Rodríguez Cabrero, 2003).

Hay que tener en cuenta que la consolidación del 0,52 no habría sido posible sin un tejido asociativo que asumiera la responsabilidad que conlleva la ejecución de esta

3 Barómetro CIS 2419. 
parte de los recursos públicos. Pero no es menos cierto que la consolidación del Tercer Sector de acción social posiblemente no hubiera sido posible de no ser por fuentes de financiación como el 0,52 las que, junto a otras fuentes de financiación públicas o privadas de diversa índole, le ha proporcionado una estabilidad financiera necesaria aunque probablemente nunca suficiente. De ahí que en dicho incremento de las entidades solicitantes del 0,52, aparte de la "innata" expansión del Tercer Sector, también haya jugado un destacado papel el incremento en los recursos destinados por este programa a otros fines de interés social y la mayor diversidad de programas de carácter prioritario contemplada en las distintas convocatorias del 0,52.

A este crecimiento del Tercer Sector de acción social le está sucediendo desde finales de los años 90 y principios del siglo XXI una etapa donde el crecimiento del número de entidades de acción social se ha estabilizado en parte por la propia madurez del sector como por el abordaje de la actividad mercantil en espacios de acción social hasta ahora circunscritos a las entidades voluntarias o por el mayor control administrativo en su colaboración con las administraciones públicas por el que tienden a desaparecer las entidades con estructuras administrativas más informales.

Estos factores, junto a que las entidades que suelen concurrir a los fondos del 0,52 son cada vez más asociaciones integradas en federaciones y confederaciones, se reflejan igualmente en la estabilidad en el número de entidades y programas subvencionados y en la evolución de las entidades solicitantes en el programa del 0,52 desde 1998. En concreto, vemos como más allá de la evolución del importe recaudado para otros fines de interés social, desde finales de los años 90 las entidades subvencionadas se han estabilizado en torno a algo más de 300 y los programas subvencionados alrededor de los 850 (Tabla 1).

Además, podemos observar en la Tabla 1 como desde 1989 hasta la actualidad los recursos destinados a otros fines de interés social gestionados por el MTAS se han más que duplicado pasando de 46 a más de 100 millones de euros, lo que ha permitido que las entidades subvencionadas y las solicitantes hayan pasado respectivamente de tan solo 37 y 96 en 1989 a más de 300 y 700 en lo que llevamos del presente siglo, o que los programas subvencionados pasen de 54 a casi 900 en dicho periodo.

Para aproximarnos a cómo el programa del 0,52 ha cubierto las expectativas de las ONG solicitantes en las distintas convocatorias podemos fijarnos en el porcentaje de los importes, entidades y programas solicitados sobre los realmente concedidos.

En cuanto al porcentaje de los programas subvencionados sobre el total de solicitados ha ido aumentando con el tiempo del $22 \%$ a principios de los años 90 a una media del $45 \%$ a principios de la década del 2000 , al igual que el porcentaje de entidades subvencionadas sobre las solicitantes que también ha aumentado del $25 \%$ a principios de los años 90 a una media del $46 \%$ desde finales de esa misma década, llegando a subvencionarse en la convocatoria IRPF 2004 a casi 5 entidades de cada 10 que lo solicitaron. Bien es cierto que parte de esta mejora se debe no solo a un incremento más moderado de las entidades del TSAS en los últimos años sino también a que las entidades que suelen concurrir a los fondos del 0,52 son cada vez más asociaciones integradas en federaciones y confederaciones. 


\begin{tabular}{|c|c|c|c|c|c|c|}
\hline \multirow{18}{*}{ 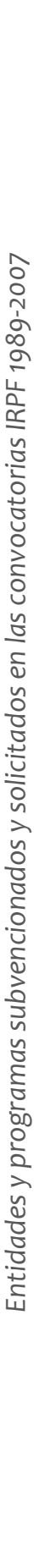 } & : & $\begin{array}{l}\infty \\
\text { ஸे } \\
\end{array}$ & $\underset{m}{\bar{q}}$ & & స్ & \\
\hline & 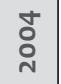 & $\begin{array}{l}\sigma \\
\dot{\sigma}\end{array}$ & $\stackrel{\bar{m}}{-}$ & $\stackrel{\circ}{\circ}$ & $\bar{\infty}$ & $\stackrel{m}{\infty}$ \\
\hline & 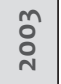 & $\tilde{n}$ & $\overline{\tilde{m}}$ & $\stackrel{\infty}{\widetilde{\sigma}}$ & ণ্ণ & $\stackrel{\stackrel{n}{N}}{\stackrel{丶}{*}}$ \\
\hline & $\begin{array}{l}\text { Õ } \\
\stackrel{D}{N}\end{array}$ & $\frac{m}{\alpha}$ & ô & 卢 & $\underset{\infty}{\tilde{\infty}}$ & $\stackrel{\infty}{\stackrel{\infty}{m}}$ \\
\hline & $\bar{\delta}$ & $\begin{array}{l}\stackrel{n}{m} \\
\tilde{\sigma}\end{array}$ & $\bar{m}$ & 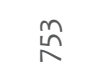 & స్ & $\begin{array}{l}\bar{\gamma} \\
\text { i }\end{array}$ \\
\hline & $\begin{array}{l}: \\
: \\
\end{array}$ & $\stackrel{+}{\sigma}$ & $\stackrel{\text { f }}{m}$ & $\stackrel{\infty}{\infty}$ & ๙̆ & $\begin{array}{l}\stackrel{\circ}{\infty} \\
\stackrel{\sim}{\sim}\end{array}$ \\
\hline & হ & $\overbrace{\infty}^{1}$ & $\stackrel{\widehat{N}}{\mathrm{~m}}$ & $\begin{array}{l}\infty \\
\infty \\
\infty\end{array}$ & $\bar{\infty}$ & 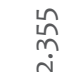 \\
\hline & $\begin{array}{l}\infty \\
\sigma \\
\sigma\end{array}$ & 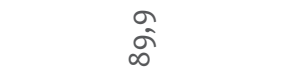 & $\stackrel{n}{m}$ & $\stackrel{\infty}{\wedge}$ & $\stackrel{\sim}{N}$ & $\frac{n}{\frac{n}{i}}$ \\
\hline & ू̆ & ñ & $\stackrel{\infty}{\sim}$ & $\stackrel{+}{N}$ & นู & $\stackrel{\infty}{\frac{m}{i}}$ \\
\hline & ஜ & $\hat{\Sigma}$ & $\stackrel{+}{\sim}$ & $\underset{\widehat{\sigma}}{\mathfrak{\gamma}}$ & $\underset{i}{J}$ & $\begin{array}{l}\stackrel{2}{2} \\
\stackrel{1}{1}\end{array}$ \\
\hline & ผू & $\stackrel{\infty}{5}$ & $\stackrel{\infty}{\infty}$ & ํㅗํ & ర্ণ & $\begin{array}{l}\circ \\
\infty \\
0\end{array}$ \\
\hline & $\begin{array}{l}\text { ஏे } \\
\text { б }\end{array}$ & 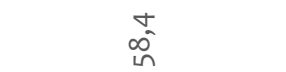 & $\stackrel{\stackrel{n}{\sim}}{=}$ & $\widehat{\widehat{\vartheta}}$ & $\bar{m}$ & 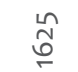 \\
\hline & & $\begin{array}{l}\text { Ln } \\
i n \\
\infty^{\circ} \\
i n\end{array}$ & $\stackrel{\circ}{\circ}$ & $\stackrel{\circ}{\aleph}$ & $\stackrel{\bar{\gamma}}{\checkmark}$ & হ \\
\hline & ๙ू & $\hat{b^{\circ}}$ & $\stackrel{+}{\stackrel{2}{*}}$ & 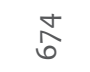 & $\bar{n}$ & $\underset{\hat{n}}{\hat{n}}$ \\
\hline & б̄ & $\stackrel{N}{\stackrel{n}{n}}$ & $\stackrel{\stackrel{\Im}{\sim}}{\sim}$ & $\hat{\hat{N}}$ & ஓे & $\stackrel{\stackrel{ }{m}}{\underset{f}{\square}}$ \\
\hline & 응 & $\hat{\tilde{y}}$ & $\bar{\infty}$ & $\stackrel{\mathscr{L}}{\sim}$ & $\underline{6}$ & ఫे \\
\hline & $\begin{array}{l}\text { ळ } \\
\text { ğ }\end{array}$ & $\stackrel{\infty}{\stackrel{\infty}{\sim}}$ & $\hat{m}$ & Һু & $\stackrel{+}{n}$ & $\stackrel{\infty}{\infty}$ \\
\hline & 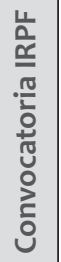 & 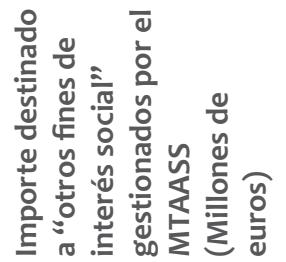 & 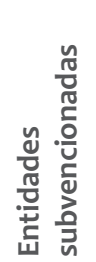 & 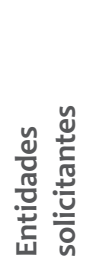 & 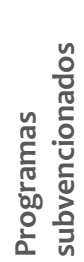 & 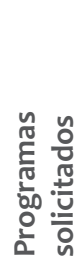 \\
\hline
\end{tabular}

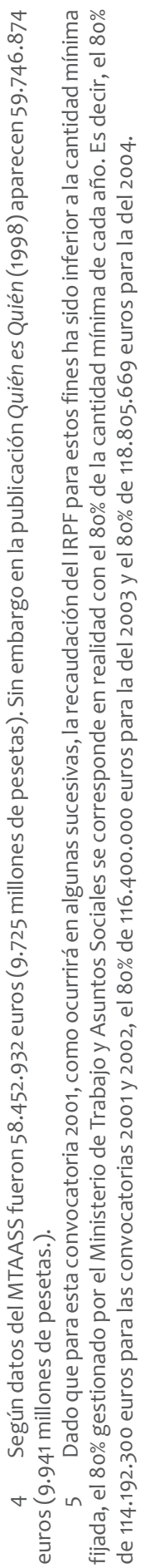



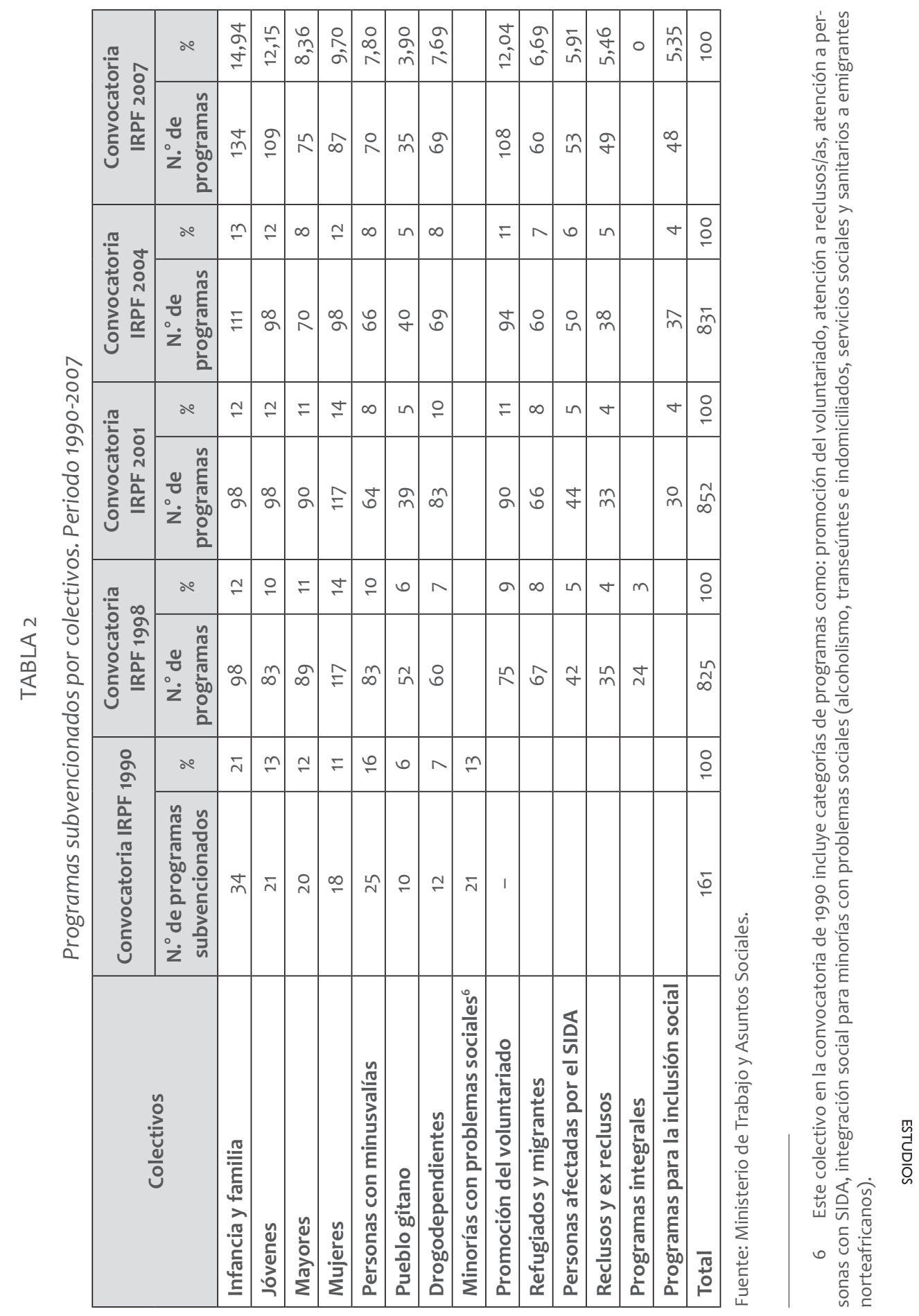


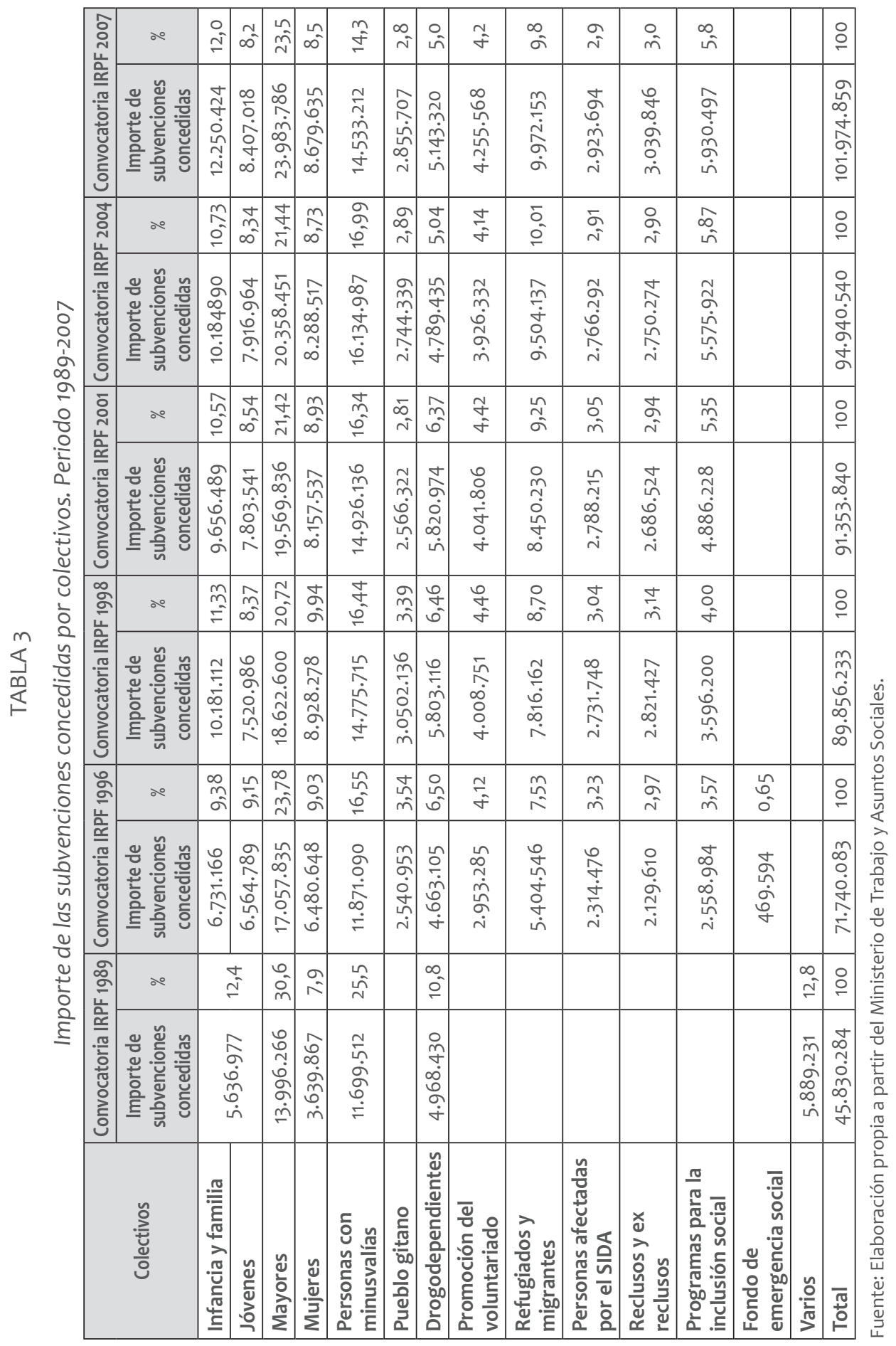




\section{EL IMPACTO SOCIOECONÓMICO DEL PROGRAMA DEL 0,52}

La consolidación del 0,52 como fuente de recursos del Tercer Sector de acción social ha reforzado la presencia de este en los espacios más recónditos de la vida social. Parece probable, no obstante, que el impacto socio-económico del 0,52 sobre el Tercer Sector ha supuesto una garantía más para que este haya alcanzado una relativa madurez institucional en el siglo XXI en la medida en la que con los requisitos exigidos a las entidades solicitantes ha contribuido a mejorar su cohesión, a fortalecer sus redes organizativas, incluso a fomentar una cultura de la gestión de calidad dentro de las ONG fruto del creciente control administrativo del que son objeto las entidades solicitantes.

Entre los impactos positivos del programa del 0,52 podríamos destacar:

1. Ha posibilitado la democratización de las decisiones fiscales y solidarias ciudadanas en las medida en la que da la oportunidad de elegir la dirección de una parte de un impuesto de carácter estatal hacia fines de interés social más allá de condicionantes territoriales. Incluso dicha democratización puede llegar a extenderse a los propios colectivos afectados quienes, aun sin llegar a ser una práctica habitual, pueden llegar a participar en los programas desarrollados por las propias entidades subvencionadas.

2. Ha supuesto una profundización en la coordinación interinstitucional e intrainstitucional. Al primer tipo ha contribuido en la medida en la que el programa del 0,52 permite ensayar modos de colaboración permanentes entre la Administración Pública y el TSAS. Al segundo porque facilita la colaboración entre entidades a través del intercambio de experiencias y porque en sus convocatorias se fomentan las redes organizativas entre las ONG de acción social y su agrupación en federaciones y confederaciones. De esta manera las ONG están aprovechando sinergias y economías de escala de gran utilidad para alcanzar con mayor éxito sus objetivos de acción social y para ampliar su radio de cobertura. Un buen ejemplo lo ilustramos con la convocatoria del 2004 en la que las 88 federaciones y confederaciones subvencionadas representaron a más de 4000 entidades de acción social. Un impacto que tiene sentido en la medida en la que el 0,52 se sustenta en el principio de interterritorialidad y cuya descentralización podría suponer un paso atrás en el fortalecimiento de ese tejido asociativo y un desmembramiento de dichas federaciones y confederaciones en la medida en la que su ámbito territorial de actuación se iría acotando.

3. Ha generado una consolidación o en su caso, el sostenimiento de las estructuras organizativas y de recursos humanos, de intervención social y de planificación estratégica de las entidades de acción social. La financiación proporcionada con del programa del 0,52 aun siendo manifiestamente insuficiente sobre todo para las de pequeño y mediano tamaño, proporciona cierta estabilidad 
en los programas desarrollados gracias a que se adelantan los fondos lo cual propicia bajos costes financieros y menores demoras en la ejecución de los programas. Esto facilita el sostenimiento de sus estructuras no solamente en términos de la dotación económica de los programas de intervención que se presentan, sino también en lo relativo a la planificación de actividades a medio plazo.

Igualmente, el programa del 0,52 está fortaleciendo el tejido asociativo no solo consolidando sus espacios de acción social, sino también sus estructuras organizativas y de recursos humanos. Por una parte, con ello podría consolidar al personal remunerado de estas entidades en la medida en la que subvenciona los gastos correspondientes a las retribuciones devengadas por el personal adscrito a las actividades desarrolladas por el programa subvencionado. Pero además puede estar favoreciendo la integración laboral dentro de las entidades y cierta estabilidad contractual. Por un lado, porque uno de los criterios de valoración de las entidades solicitantes es que en sus criterios de contratación favorezcan la integración laboral de personas con discapacidad o la de grupos sociales sobre los que existen medidas especiales de fomento del empleo, y por el otro, porque las entidades solicitantes de subvenciones para programas integrales plurianuales además deben cumplir con que el $75 \%$ del personal participante en el desarrollo de los programas esté vinculado a la entidad mediante contrato de trabajo indefinido.

4. En lo que respecta al voluntariado, aparte del propio estímulo de las entidades de acción social por incorporar voluntarios a sus estructuras organizativas, el programa del 0,52 también incentiva su promoción dentro de las organizaciones en la medida en la que prioriza a aquellas entidades y programas que cuenten con un número relevante de voluntarios y dispongan de un sistema de formación para estos. La relevancia del programa del 0,52 para el voluntariado se concreta también en los programas de promoción que se han ido subvencionando desde sus inicios llegando a ser considerado un colectivo merecedor de un programa específico de carácter prioritario. Dichos "programas de formación y/o promoción del voluntariado" han representado por término medio desde 1996 una cifra nada desdeñable del 10\% de los programas subvencionados (Tabla 2 ) y en torno al $4 \%$ de las subvenciones (Tabla 3).

5. Ha consolidado la diversidad de los colectivos a los que van dirigidas y la extensión de la intervención social hacia los colectivos más vulnerables y en riesgo de exclusión. Este carácter multisectorial se concreta en la atención de las necesidades de colectivos tan genéricos como los jóvenes, las mujeres y los mayores, las de colectivos más específicos como los afectados por discapacidades físicas, o aquellos que, independientemente de formar parte de los anteriores, padecen exclusión social por razones étnicas (gitanos, refugiados o integrales) o de otra índole (drogodependientes, enfermos de sida o reclusos). 
El impacto sobre los colectivos más precarios se observa tanto en el espectacular crecimiento de los recursos destinados a ellos como en la diversidad de grupos sociales a los que el programa del 0,52 ha ido alcanzando durante su trayectoria (Tablas 2 y 3). También adquiere especial significación tanto por su adaptabilidad a las necesidades emergentes en cada convocatoria como por el impulso que supone para los distintos Planes Nacionales como el de la Inclusión Social, el Plan Nacional de Accesibilidad, el Plan Nacional del Voluntariado, el Plan para la Igualdad de Oportunidades entre Hombre y Mujeres o el Plan Integral de Apoyo a la Familia entre otros. De hecho, la definición de los programas del 0,52 y de sus prioridades se elaboran en las distintas convocatorias del programa del 0,52 previa consulta con la Conferencia Sectorial de Asuntos Sociales y especialmente con la Comisión Permanente del Consejo Estatal de Organizaciones No Gubernamentales de Acción Social, en función de las necesidades emergentes del momento.

En cuanto a la evolución de los programas, desde el inicio del 0,52 se han ido ampliando las categorías de colectivos beneficiarios y ha ganado en concreción (Tabla 2). Con ello se pone de manifiesto la cobertura y flexibilidad del programa del 0,52 hacia la emergencia de nuevas necesidades sociales o hacia los cambios producidos en los riesgos sociales convencionales. Así se evidencia como, de las categorías de colectivos beneficiarios con la que se inició el programa (tercera edad, minusválidos, mujeres, juventud-menores, toxicómanos y varios) se ha pasado hasta las 13 actuales (infancia y familia, jóvenes, mujeres, personas mayores, personas con discapacidad, drogodependientes, pueblo gitano, migrantes y refugiados, personas afectadas por el VIH/ sida, reclusos y ex reclusos, programas para la inclusión social, promoción del voluntariado y situaciones de emergencia social). Eso ha provocado una mayor dispersión en el número de programas para los distintos colectivos, si bien a lo largo de su trayectoria prácticamente el $55 \%$ de los programas se han concentrado en cinco colectivos (infancia y familia, jóvenes, mayores, mujeres y personas con minusvalías).

En lo que respecta a los importes recibidos por cada colectivo, la dispersión es algo menor que al tratarse del número de programas. Concretamente, en los cinco colectivos mencionados anteriormente se concentran casi el $70 \%$ de las subvenciones (Tabla 3). En esta distribución por colectivos es reseñable cómo el colectivo de mayores, aunque desde el inicio de la convocatoria del 0,52 ha ido perdiendo algo de peso en el total del importe subvencionado, todavía sigue siendo el más significativo ya que a pesar de que tan solo abarca en torno a un 10\% de los programas subvencionados por término medio entre 1990 y el 2007 el importe destinado a este colectivo ha representado prácticamente el $23 \%$ de las subvenciones. De hecho, este colectivo, junto con el de discapacitados, son los que reciben importes más altos, lo cual en parte se debe al creciente envejecimiento de la población española y de las necesidades derivadas de la edad así como de la mayor tendencia de estos colectivos a coordinarse a través de federaciones y confederaciones. Tras estos colectivos, los más subvencionados durante la vigencia del $0,52 \%$ son los de infancia y familia, mujeres, jóvenes y refugiados y migrantes, lógico por otra parte dado su tamaño y relevancia social, y por último los colectivos cuantitativamente menos significativos pero con 
alto riesgo de exclusión social como drogodependientes, el pueblo gitano, reclusos y ex reclusos o personas afectadas por el SIDA.

Por otro lado, la puesta en práctica y la concreción de estos impactos aparecen de una u otra forma en los programas de las entidades subvencionadas en las últimas convocatorias del IRPF (Cuadro 1) que pueden ser una buena muestra del logro de los objetivos generales a la vez que satisfacen necesidades concretas de carácter supraterritorial. También se pone de manifiesto cómo las entidades del 0,52 unas veces actúan como pioneras en la satisfacción de ciertas necesidades sociales desarrollando innovadores programas (Red Conecta, Servicio de Información Telefónica para Embarazadas, compañías de bailes para discapacitados...), otras veces como sensores de detección de necesidades básicas y de colectivos emergentes y otras como complemento de unos servicios públicos insuficientes con los que necesita coordinarse.

\section{CUADRO 1}

Impacto del 0,52 en las necesidades sociales de los colectivos en situación de riesgo social

\begin{tabular}{|c|c|c|c|}
\hline & Colectivo & Necesidad social & Actividad/ impacto \\
\hline & Mujeres & $\begin{array}{l}\text { Mujeres maltratadas: } \\
\text { Mejorar su autoestima } \\
\text { Inserción laboral } \\
\text { Sensibilización social } \\
\text { Mujeres gestantes: } \\
\text { Prevención de } \\
\text { malformaciones } \\
\text { congénitas }\end{array}$ & $\begin{array}{l}\text { Afianzamiento de su imagen, intercambio de ex- } \\
\text { periencias con otras mujeres. Voluntariado } \\
\text { testimonial. } \\
\text { Creación y consolidación de estructuras perma- } \\
\text { nentes de inserción laboral. Individualización } \\
\text { de itinerarios de inserción: las ONG como } \\
\text { "pasarelas hacia el empleo". } \\
\text { Impartición de acciones formativas y orientación } \\
\text { para la localización y búsqueda activa de em- } \\
\text { pleo en colaboración con recursos de empleo } \\
\text { locales. } \\
\text { Campañas de sensibilización social en medios es- } \\
\text { critos y audiovisuales. } \\
\text { Asesoramiento jurídico a mujeres víctimas de la } \\
\text { violencia de género. } \\
\text { Servicios de información para prevenir factores } \\
\text { de riesgo entre embarazadas. }\end{array}$ \\
\hline & Migrantes & $\begin{array}{l}\text { Integración social, } \\
\text { acogida y asistencia } \\
\text { sociosanitaria básica. } \\
\text { Acceso a la vivienda }\end{array}$ & $\begin{array}{l}\text { Sensibilización ciudadana, promoción de la con- } \\
\text { vivencia y la interculturalidad. } \\
\text { Acogida de inmigrantes recién llegados y procura } \\
\text { de alojamiento, manutención, higiene y asis- } \\
\text { tencia sanitaria básica. } \\
\text { Posibilitar el retorno voluntario a sus países de } \\
\text { origen. } \\
\text { Intermediación en el alquiler con propietarios de } \\
\text { viviendas: mediar en conflictos, firma del con- } \\
\text { trato directamente con el propietario, avalar } \\
\text { el pago de las mensualidades... }\end{array}$ \\
\hline
\end{tabular}




$$
\text { CUADRO } 1 \text { (cont.) }
$$

Impacto del 0,52 en las necesidades sociales de los colectivos

en situación de riesgo social

\begin{tabular}{|c|c|c|}
\hline Colectivo & Necesidad social & Actividad/ impacto \\
\hline $\begin{array}{l}\text { Reclusos y ex } \\
\text { reclusos }\end{array}$ & $\begin{array}{l}\text { Reclusos y ex reclusos } \\
\text { con discapacidad: } \\
\text { Reinserción } \\
\text { sociolaboral } \\
\text { Consumo de drogas }\end{array}$ & $\begin{array}{l}\text { Creación de recursos y servicios alternativos a la } \\
\text { entrada en prisión. Unidades dependientes o } \\
\text { pisos para cumplir condena. } \\
\text { Campañas de prevención de drogas en centros } \\
\text { penitenciarios. }\end{array}$ \\
\hline Jóvenes & $\begin{array}{l}\text { Inserción laboral } \\
\text { Consumo de drogas }\end{array}$ & $\begin{array}{l}\text { Creación y consolidación de estructuras perma- } \\
\text { nentes de inserción laboral. Individualización } \\
\text { de itinerarios de inserción: las ONG como } \\
\text { "pasarelas hacia el empleo". } \\
\text { Impartición de acciones formativas y orientación } \\
\text { para la localización y búsqueda activa de em- } \\
\text { pleo en colaboración con recursos de empleo } \\
\text { locales. } \\
\text { Herramientas didácticas con alumnos de ESO } \\
\text { para la prevención de consumo de drogas. }\end{array}$ \\
\hline Voluntariado & $\begin{array}{l}\text { Formación de } \\
\text { voluntarios } \\
\text { Mayor participación } \\
\text { en la entidad }\end{array}$ & $\begin{array}{l}\text { Cursos de formación de formadores de volunta- } \\
\text { riado. } \\
\text { Elaboración de materiales de capacitación de vo- } \\
\quad \text { luntariado dirigente. } \\
\text { Encuentros de formación de voluntariado. } \\
\text { - Programas de voluntariado "testimonial". }\end{array}$ \\
\hline $\begin{array}{l}\text { Mayores y } \\
\text { familias con } \\
\text { personas } \\
\text { mayores a su } \\
\text { cargo }\end{array}$ & $\begin{array}{l}\text { Apoyo temporal y } \\
\text { servicios de respiro } \\
\text { Conciliación de vida } \\
\text { laboral y familiar }\end{array}$ & $\begin{array}{l}\text { - Servicios de teleasistencia, residencias asisti- } \\
\text { das, centros de día. } \\
\text { - Coordinación y trabajo en red con servicios } \\
\text { especializados de tercera edad, centros médi- } \\
\text { cos, Centros Municipales de Servicios Sociales } \\
\text { y con otras ONG. } \\
\text { - Adaptación de viviendas y transporte adapta- } \\
\text { do. } \\
\text { - Acompañamiento en domicilios. } \\
\text { - Información y asesoramiento sobre recursos y } \\
\text { ayudas disponibles. }\end{array}$ \\
\hline Infancia & $\begin{array}{l}\text { Infancia y } \\
\text { discapacidad: } \\
\text { Integración social } \\
\text { Erradicar los abusos } \\
\text { sexuales }\end{array}$ & $\begin{array}{l}\text { - Programas de estimulación precoz a niños dis- } \\
\text { capacitados. } \\
\text { - Cursos y seminarios de formación en preven- } \\
\text { ción de abusos sexuales a la infancia y de tu- } \\
\text { rismo sexual. }\end{array}$ \\
\hline Discapacitados & $\begin{array}{l}\text { Normalización social } \\
\text { Mejorar su autoestima }\end{array}$ & $\begin{array}{l}\text { - Ampliación de la oferta de alternativas tera- } \\
\text { péuticas, ej.: compañías de baile para discapa- } \\
\text { citados físicos, psíquicos y sensoriales. } \\
\text { - Grupos de autogestores para personas con } \\
\text { discapacidad intelectual: que "hablen por sí } \\
\text { mismas". }\end{array}$ \\
\hline
\end{tabular}


CUADRO 1 (cont.)

Impacto del 0,52 en las necesidades sociales de los colectivos

en situación de riesgo social

\begin{tabular}{|l|l|l|}
\hline \multicolumn{1}{|c|}{ Colectivo } & \multicolumn{1}{|c|}{ Necesidad social } & \multicolumn{1}{c|}{ Actividad/ impacto } \\
\hline Sida & $\begin{array}{l}\text { Normalización y } \\
\text { dignificación social }\end{array}$ & $\begin{array}{l}\text { - Pisos de acogida en las distintas fases de la } \\
\text { trayectoria vital de la enfermedad: integra- } \\
\text { ción social, cuidados en situaciones crónicas y } \\
\text { acompañamiento hasta la muerte. }\end{array}$ \\
\hline Gitanos & Integración & $\begin{array}{l}\text { - Apoyo y seguimiento de la escolarización obli- } \\
\text { gatoria de niños gitanos. }\end{array}$ \\
& $\begin{array}{l}\text { Goordinación con otras ONG, con profesores- } \\
\text { tutores, claustros y equipos de educación } \\
\text { compensatoria. }\end{array}$ \\
\hline
\end{tabular}

Fuente: Elaboración propia a partir de las memorias finales de las entidades del 0,52.

Del análisis de los programas financiados con el 0,52 se desprende que estos han contribuido a fomentar una serie de habilidades sociales como la comunicación, la escucha, el autoconocimiento y la autoestima, la tolerancia, los valores, la resolución de conflictos, la toma de decisiones y la influencia entre iguales.

No es menos importante su contribución a la ampliación de las alternativas terapéuticas ofertadas y a la atención a colectivos que se automarginan de la red sociosanitaria y asistencial pública (algunos drogodependientes con antecedentes penales, inmigrantes ilegales... ).

En cuanto al impacto del 0,52 en la satisfacción de las necesidades sociales en los distintos colectivos destacar como en relación a la inmigración están desarrollando funciones claves de intermediación con los inmigrantes en los ámbitos educativos, documental administrativo, laboral y residencial. Un buen ejemplo son los programas de acogida de inmigrantes recién llegados o la intermediación de las ONG en el alquiler de las viviendas a los propietarios donde incluso las entidades sociales llegan a avalar el pago de las mensualidades o a firmar directamente el contrato con el propietario.

En el campo de las discapacidades intelectuales están contribuyendo a superar su tradicional estigmatización y aislamiento en España no solo favoreciendo su normalización social y la mejora de su autoestima sino también ampliando la oferta de alternativas terapéuticas ya existentes.

En la atención a los mayores y a sus familias por una parte están facilitando el acceso a la información sobre los heterogéneos y a veces dispersos recursos y ayudas disponibles en el sector público, y por otra están complementando la oferta pública de servicios de respiro y de atención diurna con servicios de acompañamiento de 74 mayores en domicilios y teleasistencia, con la gestión de sus propios centros en cola- 
boración con las Comunidades Autónomas y Administraciones locales (residencias, centros de día).

Para colectivos susceptibles de verse especialmente afectados por la exclusión social y laboral (inmigrantes, mujeres maltratadas, ex reclusos, personas con sida...) se están potenciando actividades de sensibilización social y consolidando estructuras permanentes de inserción laboral individualizando itinerarios de inserción.

En el caso del voluntariado, con los programas subvencionados por el 0,52 no solo se estimula la entrada de voluntarios sino también su formación como futuros formadores o como voluntarios dirigentes.

Más allá de las bondades descritas, el programa del 0,52 también adolece de una serie de limitaciones especialmente en lo que respecta a la dotación de recursos, a la continuidad de los programas o a la agilidad administrativa en los procesos de solicitud.

1. En particular subrayaríamos que, aunque ya antes de la acertada introducción en el año 2005 de los programas plurianuales la mayoría de las subvenciones eran para programas anuales pero con cierta continuidad de los mismos, lo que permitía una plurianualidad de facto, sucede que siguen siendo muy minoritarios en el del 0,52 (González y de Cabo, 2008) debido a la dificultad de cumplir con alguno de los requisitos (como que el $75 \%$ del personal participante en el desarrollo de los programas tenga un contrato de trabajo indefinido); a una residual dotación económica ya que solo puede destinarse a ellos el $2 \%$ del crédito total disponible; y a su finalidad selectiva pues solo se conceden para programas destinados a personas en riesgo de exclusión social y a personas dependientes mayores o discapacitadas.

2. Otra de las limitaciones del programa son los elevados costes administrativos y burocráticos que suponen el cumplimiento económico-administrativo, de control y de evaluación de resultados de los proyectos por parte de la Administración, sobre todo para algunas entidades cuyos medios apenas les alcanzan para elaborar y ejecutar sus propios programas.

3. Por último, la multitud de colectivos de intervención a los que atiende y en los que está organizado el programa del 0,52 a veces va en detrimento de otros objetivos transversales y de intervención múltiple como pueden ser los programas preventivos, probablemente por la difícil estimación de su rentabilidad social en el corto plazo.

\section{CONCLUSIONES}

El desarrollo de las entidades de acción social en los últimos años, en especial en su vertiente más prestacional, se ha visto fortalecido por los programas de subven- 
ciones de las distintas administraciones públicas. Un buen ejemplo es el programa del 0,52, o del 0,7 desde la convocatoria del IRPF de 2009, al que consideramos como un programa singular en nuestro Estado Autonómico tanto por su larga vigencia como por su ámbito territorial (nacional) y al que concurren la gran parte de las entidades de acción social bajo modos organizativos federales.

El programa del 0,52 está contribuyendo a mejorar la situación de los colectivos en situación de riesgo social y a consolidar en unos casos, y a sostener en otros, las estructuras organizativas en varios ámbitos.

En el ámbito territorial, al tratarse de un programa de alcance nacional está contribuyendo a fomentan las redes organizativas entre entidades sociales de distintas Comunidades Autónomas y a profundizar en la coordinación interinstitucional e intrainstitucional de las organizaciones. Esto está propiciando una mayor coordinación de acciones entre las ONG y la generación de políticas globales que de otra manera difícilmente podrían desarrollarse en el actual sistema de competencias en materia de política social.

En el ámbito de los recursos humanos, la dotación económica del programa, aunque claramente insuficiente, ha sido creciente en los últimos años y ello está contribuyendo al sostenimiento de las estructuras organizativas y de recursos humanos, de intervención social y de planificación estratégica de las entidades de acción social. Está permitiendo multiplicar recursos convergentes para una misma acción o programa.

No obstante, el programa adolece de una serie de limitaciones (lentitud administrativa, insuficiente dotación, escasa plurianualidad de las subvenciones y la debilidad de las acciones transversales, preventivas y promocionales de los programas de intervención social) que aconsejan una paulatina reforma. En particular, las reformas deberían ir encaminadas a: 1) consolidar y reforzar los medios económicos en proyectos de carácter transversal, plurianual y preventivo; 2) a agilizar los procesos administrativos, por ejemplo, mejorando los sistemas de información entre los financiadores públicos y privados con el fin de evitar que las entidades subvencionadas tengan que justificar reiteradamente los recursos que reciben para un mismo programa; 3) potenciar la visibilidad social de las entidades subvencionadas con cargo al programa del 0,52; y 4) mejorar los sistemas de evaluación y seguimiento del cumplimiento de los objetivos establecidos en el programa subvencionado, así como elaborar un sistema de indicadores que evalúen la calidad de la provisión de los servicios de las entidades subvencionadas. 


\section{REFERENCIAS BIBLIOGRÁFICAS Y DOCUMENTALES}

De la Fuente, C. y Montraveta, I. (2002), Quien es quién: Convocatoria 2001. Madrid: Ministerio de Trabajo y Asuntos Sociales.

De la Fuente, C., Montraveta, I. y Simon, C. (2005), Quien es quién: Convocatoria 2004. Madrid: Ministerio de Trabajo y Asuntos Sociales.

De la Fuente, C., Montraveta, I. y Valls, R. (1999), Quien es quién: Convocatoria 1998. Madrid: Ministerio de Trabajo y Asuntos Sociales.

García Delgado, J.L dir. (2004), Las Cuentas de la Economía Social. El Tercer Sector en España. Madrid: Civitas.

González, E. y De Cabo, G. coord. (2008), Quien es quién. Las entidades de acción social beneficiarias de la asignación tributaria del IRPF. Madrid: Ministerio de Trabajo y Asuntos Sociales.

Pérez Díaz, V. y López Novo, J. P. (2003), El Tercer Sector Social en España. Madrid: Ministerio de Trabajo y Asuntos Sociales.

Rodríguez Cabrero, G. y Montserrat, J. (1996), Las entidades voluntarias en España. Madrid: Ministerio de Asuntos Sociales.

Rodríguez Cabrero, G., coord., (2003), Las entidades voluntarias de acción social en España. Madrid: Fundación FOESSA.

Salamon, L. M., et al. (2001), La Sociedad Civil Global. Las dimensiones del sector no lucrativo. Bilbao: Fundación BBVA.

Salinas, F., Rubio, M. J. y Cerezo, I. (2001), La evolución del Tercer Sector hacia la Empresa Social. Madrid: Plataforma para la Promoción del Voluntariado en España.

VV.AA. (2000), Empleo y trabajo voluntario en las ONGs de acción social. Madrid: Fundación Tomillo.

VV.AA. (1997), Las Organizaciones de Voluntariado en España. Documento de trabajo n. ${ }^{\circ}$ 10. Madrid: Plataforma para la promoción del voluntariado en España (PPVE). 